\title{
Ventriculoatrial Block During a Narrow-QRS Tachycardia: What Is the Tachycardia Mechanism?-IV
}

\author{
FRED MORADY, M.D. \\ From the Cardiology Division, Department of Internal Medicine, \\ University of Michigan Medical Center, Ann Arbor, Michigan
}

\section{Case Presentation}

A 28-year-old man underwent an electrophysiologic procedure because of frequent episodes of paroxysmal supraventricular tachycardia. The baseline sinus cycle length, atrial-His $(\mathrm{AH})$ interval, and His-ventricular (HV) intervals were 800 , 90 , and $40 \mathrm{msec}$, respectively. A narrow-QRS tachycardia having a cycle length of 250 to 280 msec was reproducibly induced by ventricular programmed stimulation with a single extrastimulus (Figs. 1 and 2). There was an inverse relationship between the coupling interval of the ventricular extrastimulus that induced the tachycardia and the interval between the extrastimulus and the first tachycardia complex. The HV interval during tachycardia was $40 \mathrm{msec}$. A ventricular premature depolarization introduced during the tachycardia simultaneous with the His-bundle depolarization did not reset or terminate the tachycardia. The tachycardia was consistently terminated by ventricular pacing at a cycle length of $230 \mathrm{msec}$. What is the mechanism of this tachycardia?

\section{Commentary}

The interesting and unusual aspect of this narrow-QRS tachycardia is that it was inducible by ventricular pacing without depolarization of the atrium and that there was a $2: 1$ relationship between the ventricle and right atrium during the tachycardia. Figure 1 demonstrates that the ven-

J Cardiovasc Electrophysiol, Vol. 7, pp. 174-177, February 1996.

Address for correspondence: Fred Morady, M.D., University of Michigan Medical Center, 1500 East Medical Center Drive, B1F245, Ann Arbor, MI 48109-0022. Fax: 313-936-7026.

Manuscript received 22 November 1995; Accepted for publication 22 November 1995. tricular depolarizations during the basic drive train resulted in retrograde conduction to the atrium, but that the ventricular extrastimulus that induced the tachycardia did not result in an atrial depolarization. As can be seen in Figure 2, every other ventricular electrogram during the tachycardia is followed by an atrial electrogram.

The fact that induction of the tachycardia did not require depolarization of the atrium immediately rules out the possibility of an atrial tachycardia. Along with the 1:2 AV relationship during the tachycardia, this also rules out the possibility of orthodromic tachycardia utilizing a concealed accessory pathway as its retrograde limb. By exclusion, this tachycardia must be arising in or below the AV node. The differential diagnosis includes an automatic junctional tachycardia, AV nodal reentrant tachycardia, a tachycardia caused by intra-Hisian reentry, and a reentrant tachycardia using the AV junction as the anterograde limb of the reentry circuit and a concealed nodoventricular or nodofascicular pathway as the retrograde limb. Any of these mechanisms might account for a narrow-QRS tachycardia with 2:1 retrograde block to the atrium.

Initiation and termination of the tachycardia by pacing maneuvers would not be expected in an automatic junctional tachycardia. Although a junctional tachycardia caused by triggered activity would be induced and terminated by pacing maneuvers, the reciprocal relationship between the coupling interval of the ventricular extrastimulus that induced the tachycardia and the interval between the extrastimulus and the first tachycardia complex strongly favors a reentrant mechanism.

The differential diagnosis can be narrowed down further by assessing the response to a ventricular premature depolarization introduced during the tachycardia simultaneous with the His-bundle 

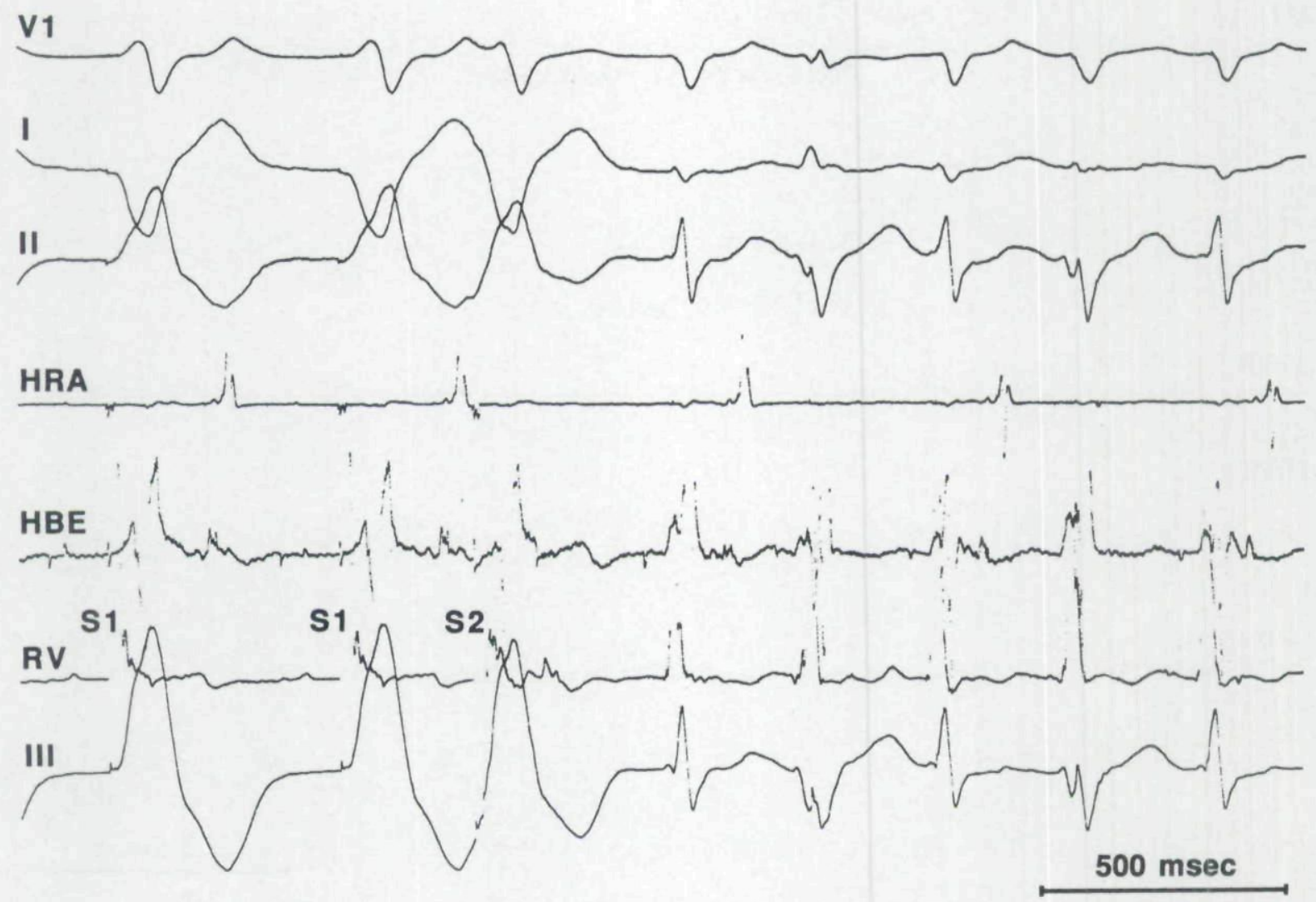

Figure 1. Induction of tachycardia by programmed ventricular stimulation with a single extrastimulus (S2). From top to bottom are leads VI, I, and II, the high right atrial electrogram (HRA), His-bundle electrogram (HBE), right ventricular electrogram (RV), and lead III. The tachycardia cycle length is 270 to $275 \mathrm{msec}$.

depolarization. In AV nodal reentrant tachycardia, a ventricular premature depolarization simultaneous with the His-bundle depolarization would not be able to penetrate the reentry circuit. In contrast, in a tachycardia caused by intra-Hisian reentry or in a reentrant tachycardia using the AV junction as the anterograde limb of the reentry circuit and a concealed nodoventricular or nodofascicular pathway as the retrograde limb, a ventricular premature depolarization simultaneous with the His-bundle depolarization should be able to penetrate the reentry circuit and either reset or terminate the tachycardia. Therefore, in this case, the inability of a ventricular premature depolarization simultaneous with the His-bundle potential to affect the tachycardia favors the diagnosis of AV nodal reentrant tachycardia. However, other lines of evidence that usually are available to support the diagnosis of $\mathrm{AV}$ nodal reentrant tachycardia (e.g., the requirement for a critical $\mathrm{AH}$ interval to induce the tachycardia or the response of the tachycardia to atrial and ventricular pacing) are lacking in this case, either because the tachycardia was inducible only by ventricular pacing or because of the $1: 2 \mathrm{AV}$ relationship.

The intriguing possibility of intra-Hisian reentrant tachycardia deserves further consideration. First of all, it must be acknowledged that the inability of a ventricular premature depolarization simultaneous with the His-bundle potential to affect the tachycardia does not rule out intra-Hisian reentrant tachycardia. Second, although one might expect to see evidence of longitudinal dissociation in the His bundle, i.e., two different HV intervals during atrial pacing, the absence of this finding does not rule out intra-Hisian reentrant tachycardia, just as the absence of dual AV nodal physiology does not rule out AV nodal reentrant tachycardia. Third, in intra-Hisian reentrant tachycardia, two His-bundle potentials should be present for every ventricular electrogram, one generated in the retrograde limb of the reentry circuit and the other in the anterograde limb. Figure 1 demonstrates that the first ventricular complex of the tachycardia is, in fact, preceded by two His-bundle electrograms: one that immediately follows the ventricular 

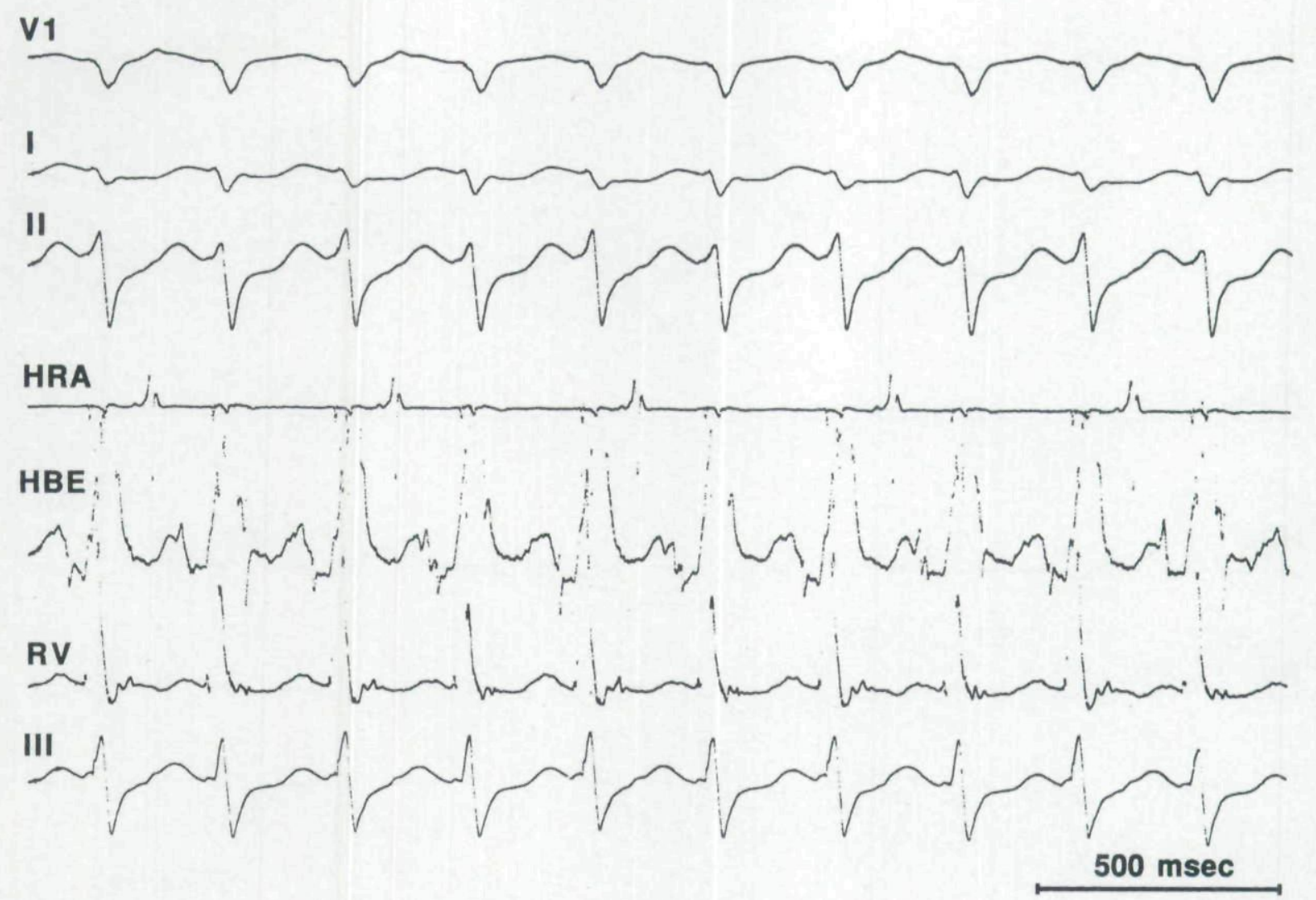

Figure 2. An example of the tachycardia several seconds after its induction. The tachycardia cycle length is $250 \mathrm{msec}$, Abbreviations as in Figure 1 .

electrogram generated by $\mathrm{S} 2$, and a second one that is $40 \mathrm{msec}$ in front of the ventricular electrogram of the first tachycardia complex. During the tachycardia, only one His-bundle potential is clearly seen, but it may be that there is another one buried in the latter half of the ventricular electrograms. A tantalizing hint of this possibility is present in the second, eighth, and tenth ventricular complexes in Figure 2, in which there is a high-frequency deflection suggestive of a His-bundle potential at the tail end of the ventricular potentials.

Because the His bundle is not part of the AV nodal reentrant tachycardia circuit, in theory it should be possible to dissociate the His-bundle potential from the tachycardia. In contrast, in intraHisian reentrant tachycardia, it would not be possible to dissociate the His-bundle potential from the tachycardia. Therefore, if the His-bundle potential could be dissociated from the tachycardia, this would provide definitive evidence against intra-Hisian reentrant tachycardia. However, dissociation of the His potential from the tachycardia in this case would have required His-bundle pacing, which is difficult to achieve without ventric- ular capture and which was not attempted.

The tachycardia was successfully eliminated by radiofrequency slow pathway ablation during sinus rhythm in the posteroseptal right atrium at the level of the coronary sinus ostium, where no Hisbundle potential was recorded. This provides strong evidence that the tachycardia was AV nodal reentrant tachycardia as opposed to intra-Hisian reentrant tachycardia. Were it not for the response to ablation, the definitive exclusion of intra-Hisian reentrant tachycardia may not have been possible in this case.

An incidental observation in Figure 1 is that the second and fourth tachycardia complexes display left bundle branch block aberration. This suggests that the ventricular depolarization caused by $\mathrm{S} 2$ resulted in retrograde block in the left bundle and conduction to the His bundle through the right bundle. This would set up a "long-short" sequence in the left bundle, accounting for functional block.

A final point worthy of comment has to do with the mechanistic implications of VA block during AV nodal reentrant tachycardia. The occurrence of 
VA block during the tachycardia provides compelling evidence that the AV nodal reentry circuit has an upper common pathway within the AV node. On the other hand, evidence from high-density electrode mapping and ice-mapping studies in the operating room, the response to entrainment, and the efficacy of surgical and radiofrequency ablation techniques directed at perinodal atrial inputs to the AV node seem to indicate that there is no upper common pathway and that the perinodal atrium is part of the AV nodal reentrant tachycardia circuit. Perhaps these contradictory lines of evidence are explained by the existence of more than one type of AV nodal reentrant tachycardia. Even so, what remains puzzling in the present case is why radiofrequency ablation at the ostium of the coronary sinus was able to eliminate a tachycardia that was presumably limited to the AV node. It is clear that our understanding of AV nodal reentrant tachycardia remains incomplete. 
This document is a scanned copy of a printed document. No warranty is given about the accuracy of the copy. Users should refer to the original published version of the material. 